\title{
Remarks on Convolutions and Fractional Derivative of Distributions
}

\author{
Chenkuan $\mathrm{Li}^{1} \&$ Kyle Clarkson ${ }^{1}$
}

${ }^{1}$ Department of Mathematics and Computer Science, Brandon University, Brandon, Manitoba Canada R7A 6A9

Correspondence: Chenkuan Li, Department of Mathematics and Computer Science, Brandon University, Brandon, Manitoba Canada R7A 6A9. Tel: 204-571-8549. E-mail: lic@ brandonu.ca

Received: October 25, 2017 Accepted: November 6, 2017 Online Published: November 17, 2017

doi:10.5539/jmr.v10n1p6

URL: https://doi.org/10.5539/jmr.v10n1p6

\section{Abstract}

This paper begins to present relations among the convolutional definitions given by Fisher and $\mathrm{Li}$, and further shows that the following fractional Taylor's expansion holds based on convolution

$$
\frac{d^{\lambda}}{d x^{\lambda}} \theta(x) \phi(x)=\sum_{k=0}^{\infty} \frac{\phi^{(k)}(0) x_{+}^{k-\lambda}}{\Gamma(k-\lambda+1)} \quad \text { if } \quad \lambda \geq 0,
$$

with demonstration of several examples. As an application, we solve the Poisson's integral equation below

$$
\int_{0}^{\pi / 2} f(x \cos \omega) \sin ^{2 \lambda+1} \omega d \omega=\theta(x) g(x)
$$

by fractional derivative of distributions and the Taylor's expansion obtained.

Keywords: Distribution, Convolution, Fractional Taylor's expansion, Neutrix limit, Fractional derivative, Stirling's formula.

\section{Introduction}

One of the serious challenges in distribution theory is how to define convolution and product of distributions in general (Fisher, 1987, Cheng \& Li, 1991; Li, 2005; Aguirre \& Li, 2007; Li, 2007a; Li, 2007b). It is well known that studying fractional calculus of distributions and many types of integral equations are based on distributional convolutions (Gel'fand \& Shilov, 1964; Kilbas, Srivastava, \& Trujillo, 2006; Li \& Li, 2014; Li, 2015; Li \& Li, 2017; Li, Li, Kacsmar, Lacroix, $\&$ Tilbury, 2017). There have been several suggested definitions for distributional convolution, each of which generalizes the following classical definition for the convolution of two functions $f$ and $g$, given as follows:

Definition 1.1 Let $f$ and $g$ be functions. Then the convolutional product $f * g$ is defined by

$$
(f * g)(x)=\int_{-\infty}^{\infty} f(t) g(x-t) d t
$$

if the integral exists.

Let $\mathcal{D}$ be the Schwartz space (Gel'fand \& Shilov, 1964) of infinitely differentiable functions with compact support in $R$, and $\mathcal{D}^{\prime}$ be the space of distributions (linearly continuous functionals) defined on $\mathcal{D}$. Further, we shall define a sequence $\phi_{1}(x), \phi_{2}(x), \cdots, \phi_{n}(x), \cdots$ which converges to zero in $\mathcal{D}$ if all these functions vanish outside a certain fixed and bounded interval, and converge uniformly to zero (in the usual sense) together with their derivatives of any order. The functional $\delta$ is defined as

$$
(\delta, \phi)=\phi(0)
$$

where $\phi \in \mathcal{D}$. Clearly, $\delta$ is a linear and continuous functional on $\mathcal{D}$, and hence $\delta \in \mathcal{D}^{\prime}$.

The convolution of certain pairs of distributions is usually defined as follows, see (Gel'fand \& Shilov, 1964) for example.

Definition 1.2 Let $f$ and $g$ be distributions in $\mathcal{D}^{\prime}$ satisfying either of the following conditions:

(a) either $f$ or $g$ has bounded support (set of all essential points), or

(b) the supports of $f$ and $g$ are bounded on the same side.

Then the convolution $f * g$ is defined by the equation

$$
((f * g)(x), \phi(x))=(g(x),(f(y), \phi(x+y)))
$$


for $\phi \in \mathcal{D}$.

Note that if $f$ and $g$ are locally integrable functions satisfying either of the conditions in (a) or (b) in Definition 1.2, then Definition 1.2 is in agreement with Definition 1.1. It also follows that if the convolution $f * g$ exists by Definition 1.1 or 1.2 , then the following equations hold:

$$
\begin{aligned}
& f * g=g * f \\
& (f * g)^{\prime}=f * g^{\prime}=f^{\prime} * g
\end{aligned}
$$

where all the derivatives above are in the distributional sense.

Both Definitions 1.1 and 1.2 are very restrictive and can only be used for a small class of distributions. In order to extend the convolution to a larger class of distributions, (Jones, 1973) introduced the following definition:

Definition 1.3 Let $f$ and $g$ be distributions in $\mathcal{D}^{\prime}$ and let $\tau(x)$ be an infinitely differentiable function satisfying the following conditions:

(i) $\tau(x)=\tau(-x)$,

(ii) $0 \leq \tau(x) \leq 1$,

(iii) $\tau(x)=1$ if $|x| \leq 1 / 2$,

(iv) $\tau(x)=0$ if $|x| \geq 1$.

Let

$$
f_{n}(x)=f(x) \tau(x / n), \quad g_{n}(x)=g(x) \tau(x / n)
$$

for $n=1,2, \cdots$. Then the convolution $f * g$ is defined as the limit of the sequence $\left\{f_{n} * g_{n}\right\}$, provided the limit $h$ exists in the sense that

$$
\lim _{n \rightarrow \infty}\left(f_{n} * g_{n}, \phi\right)=(h, \phi)
$$

for all testing functions $\phi \in \mathcal{D}$.

The convolution $f_{n} * g_{n}$ in Definition 1.3 clearly exists by Definition 1.2 as $f_{n}$ and $g_{n}$ have bounded support. Furthermore, equation (1) holds. However, equation (2) need not necessarily hold since (Jones, 1973) proved that

$$
\begin{aligned}
& 1 * \operatorname{sgn} x=\operatorname{sgn} x * 1=x, \\
& (1 * \operatorname{sgn} x)^{\prime}=1,1^{\prime} * \operatorname{sgn} x=0,1 *(\operatorname{sgn} x)^{\prime}=1 * 2 \delta(x)=2 .
\end{aligned}
$$

It can be proven that if a convolution exists by Definition 1.1 or 1.2 then it exists by Definition 1.3 and defines the same distribution. Therefore, Definition 1.3 generalizes Definitions 1.1 and 1.2. However, there are still many convolutions which cannot be given by Definition 1.3. In order to fix this, (Fisher, 1987) presented the following definition:

Definition 1.4 Let $f$ and $g$ be distributions and let

$$
\tau_{n}(x)= \begin{cases}1 & \text { if }|x| \leq n, \\ \tau\left(n^{n} x-n^{n+1}\right) & \text { if } x>n, \\ \tau\left(n^{n} x+n^{n+1}\right) & \text { if } x<-n,\end{cases}
$$

for $n=1,2, \cdots$, where $\tau$ is defined as in Definition 1.3. Let $f_{n}(x)=f(x) \tau_{n}(x)$ for $n=1,2, \cdots$. Then the noncommutative neutrix convolution $f \odot g$ is defined as the neutrix limit of the sequence $\left\{f_{n} * g\right\}$, provided the limit $h$ exists in the sense that

$$
N-\lim _{n \rightarrow \infty}\left(f_{n} * g, \phi\right)=(h, \phi)
$$

for all $\phi \in \mathcal{D}$, where $N$ is the neutrix, (van der Corput, 1959-60) (use the neutrix to abandon unwanted infinite quantities from asymptotic expressions), having domain $N^{\prime}=\{1,2, \cdots\}$ and range the real numbers, with negligible functions that are finite linear sums of functions

$$
n^{\lambda} \ln ^{r-1} n, \quad \ln ^{r} n, \quad(\lambda>0, r=1,2, \cdots)
$$

and all functions of $n$ that converge to zero in the normal sense as $n$ tends to infinity. 
The convolution $f_{n} * g$ in this definition is again in the sense of Definition 1.2 as the support of $f_{n}$ is contained in the interval $\left[-n-n^{-n}, n+n^{-n}\right]$. It is also proven that if a convolution exists by Definition 1.1 or 1.2 then the noncommutative neutrix convolution exists and defines the same distribution (Fisher, 1987).

To overcome the convolutional noncommutativity above, (Fisher \& Li, 1993) introduced the following commutative neutrix convolution of distributions $f$ and $g$ by $f \otimes g$ to distinguish it from the noncommutative neutrix convolution in Definition 1.4:

Definition 1.5 Let $f$ and $g$ be distributions and let $\tau_{n}$ be defined as in Definition 1.4. Let $f_{n}(x)=f(x) \tau_{n}(x)$ and $g_{n}(x)=$ $g(x) \tau_{n}(x)$ for $n=1,2, \cdots$. Then the commutative neutrix convolution $f \otimes g$ is defined as the neutrix limit of the sequence $\left\{f_{n} * g_{n}\right\}$, provided the limit $h$ exists in the sense that

$$
N-\lim _{n \rightarrow \infty}\left(f_{n} * g_{n}, \phi\right)=(h, \phi)
$$

for all $\phi \in \mathcal{D}$, where $N$ is the neutrix given above.

While Definition 1.5 defines the commutative neutrix convolution, its computational complexity hinders the calculation processes due to the factor $\tau_{n}(t) \tau_{n}(x-t)$ appearing in the convolution $f_{n} * g_{n}$. To address this, (Li, Clarkson, \& Patel, in press) recently introduced the following definition:

Definition 1.6 Let $f$ and $g$ be distributions and let $\tau_{n}$ be defined as in Definition 1.4. Let $f_{n}(x)=f(x) \tau_{n}(x)$ and $g_{n}(x)=$ $g(x) \tau_{n}(x)$ for $n=1,2, \cdots$. Then the commutative neutrix convolution $f * g$ of $f$ and $g$ is defined as the neutrix limit of the sequence $1 / 2\left\{f_{n} * g+f * g_{n}\right\}$, provided the limit $h$ exists in the sense that

$$
N-\lim _{n \rightarrow \infty} \frac{1}{2}\left\{\left(f_{n} * g, \phi\right)+\left(f * g_{n}, \phi\right)\right\}=(h, \phi)
$$

for all $\phi \in \mathcal{D}$. If the normal limit exists, then it is simply called the commutative convolution. Clearly, this definition generalizes Definitions 1.1 and 1.2.

Notes on the neutrix limit: (Fisher, 1982), with his coauthors (Fisher \& Kuribayashi, 1987; Fisher \& Taş, 2005; Fisher \& Ozcag, 2012; Fisher \& Al-Sirehy, 2015; Lazarova, Jolevska-Tuneska, Akturk, \& Ozcag, 2016; Ozcag, Lazarova, \& Jolevska-Tuneska, 2016; Fisher, Ozcag, \& Al-Sirehy, 2017), has actively used Temples' $\delta$-sequence and the concept of neutrix limit to deduce numerous products, powers, convolutions, and compositions of distributions. The technique of neglecting appropriately defined infinite quantities and resulting in a finite value extracted from the divergent integral, is usually referred to as the Hadamard finite part. In fact, Fisher's method in the computation can be regarded as a particular application of the neutrix calculus. This is a general principle for the discarding of unwanted infinite quantities from asymptotic expansions and has been exploited in the context of distribution by Fisher in connection with the problem of distributional multiplication, convolution and composition.

On the other hand, fractional calculus, first mentioned in the letter from Leibniz to L'Hôpital dated 30 September 1695, can be regarded as a branch of analysis which deals with integral and differential equations often with weakly singular kernels. A lot of contributions to the theory of fractional calculus up to the middle of the 20th century were made by many famous mathematicians including Laplace, Fourier, Abel, Liouville, Riemann, Grünwald, Letnikov, Heaviside, Weyl, Erdélyi and others. After 1970, there was a clear movement from theoretical research of fractional calculus to its applications in various fields. Up to now, fractional calculus has been found in almost every realm of science and engineering. As far as we know, it is one of the best tools to characterize long-memory processes and materials, anomalous diffusion, long-range interactions, long-term behaviors, power laws, allometric scaling laws, and so on.

As outlined in the abstract, the goal of this paper is to discuss relations among different convolutional definitions and define fractional derivatives and integrals based on Definition 1.6. We further present the fractional Taylor's expansion for the distribution $\frac{d^{\lambda}}{d x^{\lambda}} \theta(x) \phi(x)$ as well as its applications to solving several Poisson's integral equations and a one-term differential equation in distribution.

\section{Remarks on the Convolutions}

It seems true that Definition 1.5 is equivalent to Definition 1.6 from many examples calculated in (Li et al., in press) and the following argument:

$$
\begin{aligned}
& N-\lim _{n \rightarrow \infty} \frac{1}{2}\left\{\left(\left(f \tau_{n}\right) * g, \phi\right)+\left(f *\left(g \tau_{n}\right), \phi\right)\right\}-N-\lim _{n \rightarrow \infty}\left(f_{n} * g_{n}, \phi\right) \\
& =N-\lim _{n \rightarrow \infty} \frac{1}{2}\left\{\left(\left(f \tau_{n}\right) *\left(g-g \tau_{n}\right), \phi\right)+\left(\left(f-f \tau_{n}\right) *\left(g \tau_{n}\right), \phi\right)\right\} .
\end{aligned}
$$


Our first result is stated in Theorem 2.1 below, where we prove the opposite by a counterexample.

Theorem 2.1 Definition 1.5 is not equivalent to Definition 1.6.

Proof. By Definition 1.5, we have

$$
(x \otimes 1, \phi(x))=N-\lim _{n \rightarrow \infty}\left(x \tau_{n}(x) * \tau_{n}(x), \phi(x)\right)
$$

and

$$
\begin{aligned}
x \tau_{n}(x) * \tau_{n}(x) & =\int_{-\infty}^{\infty} t \tau_{n}(t) \tau_{n}(x-t) d t \\
& =\int_{-n-n^{-n}}^{-n} t \tau_{n}(t) \tau_{n}(x-t) d t+\int_{-n}^{n} t \tau_{n}(x-t) d t+\int_{n}^{n+n^{-n}} t \tau_{n}(t) \tau_{n}(x-t) d t \\
& =I_{1}+I_{2}+I_{3} .
\end{aligned}
$$

It follows that

$$
\lim _{n \rightarrow \infty} I_{1}=\lim _{n \rightarrow \infty} I_{3}=0
$$

by noting that $\tau_{n}(t) \tau_{n}(x-t)$ is bounded.

Making the substitution $u=x-t$, we get

$$
I_{2}=\int_{x-n}^{x+n}(x-u) \tau_{n}(u) d u
$$

For $x>0$, choose $n$ such that $n^{-n}<x$, then

$$
I_{2}=\int_{x-n}^{n}(x-u) d u+\int_{n}^{n+n^{-n}}(x-u) \tau_{n}(u) d u=\left.\left(x u-\frac{u^{2}}{2}\right)\right|_{x-n} ^{n}+\int_{n}^{n+n^{-n}}(x-u) \tau_{n}(u) d u .
$$

It follows that for $x>0$,

$$
N-\lim _{n \rightarrow \infty}\left(I_{2}, \phi\right)=\left(-\frac{x^{2}}{2}, \phi(x)\right)
$$

For $x<0$, choose $n$ such that $-n^{-n}>x$, then

$$
I_{2}=\int_{-n-n^{-n}}^{-n}(x-u) \tau_{n}(u) d u+\int_{-n}^{x+n}(x-u) d u=\int_{-n-n^{-n}}^{-n}(x-u) \tau_{n}(u) d u+\left.\left(x u-\frac{u^{2}}{2}\right)\right|_{-n} ^{x+n} .
$$

It follows that for $x<0$,

$$
N-\lim _{n \rightarrow \infty}\left(I_{2}, \phi\right)=\left(\frac{x^{2}}{2}, \phi(x)\right)
$$

Therefore,

$$
x \otimes 1=-\frac{x}{2}|x|
$$

By Definition 1.6, we have

$$
(x \approx 1, \phi(x))=N-\lim _{n \rightarrow \infty} \frac{1}{2}\left\{\left(x \tau_{n}(x) * 1, \phi(x)\right)+\left(x * \tau_{n}(x), \phi(x)\right)\right\} .
$$

We first notice that $x \tau_{n}(x) * 1$ evaluates to zero, as the resulting integrand is an odd function, that is,

$$
x \tau_{n}(x) * 1=\int_{-\infty}^{\infty} t \tau_{n}(t) d t=0 .
$$

As for $x * \tau_{n}(x)$, by substituting $u=x-t$, we have

$$
\begin{aligned}
& \int_{-\infty}^{\infty} t \tau_{n}(x-t) d t=\int_{-\infty}^{\infty}(x-u) \tau_{n}(u) d u \\
& =x \int_{-\infty}^{\infty} \tau_{n}(u) d u-\int_{-\infty}^{\infty} u \tau_{n}(u) d u=I_{1}+I_{2} .
\end{aligned}
$$


Again, it is clear that $I_{2}$ evaluates to zero. For $I_{1}$, we have

$$
I_{1}=x \int_{-n-n^{-n}}^{-n} \tau_{n}(u) d u+x \int_{-n}^{n} d u+\int_{n}^{n+n^{-n}} \tau_{n}(u) d u .
$$

With the use of the neutrix limit,

$$
N-\lim _{n \rightarrow \infty}\left(x * \tau_{n}(x), \phi(x)\right)=N-\lim _{n \rightarrow \infty}(2 x n, \phi(x))=0 .
$$

Thus,

$$
x \bar{*} 1=0
$$

by Definition 1.6. This completes the proof of Theorem 2.1.

Remark 1 It is interesting to point out that if we add the neutrix limit in Definition 1.3 then the convolution $x * 1$ is also zero (otherwise, it diverges). In fact,

$$
(x * 1, \phi)=N-\lim _{n \rightarrow \infty}(x \tau(x / n) * \tau(x / n), \phi) .
$$

Evidently,

$$
x \tau(x / n) * \tau(x / n)=n^{2} \int_{-1}^{1} y \tau(y) \tau(x / n-y) d y .
$$

By the mean value theorem,

$$
\lim _{n \rightarrow \infty} n \int_{-1}^{1} y \tau(y) \tau(x / n-y) d y=-x \int_{-1}^{1} \tau^{\prime}(y) y \tau(y) d y .
$$

for $x$ in the support of $\phi$. Choosing $\phi$ such that $\int_{-\infty}^{\infty} x \phi(x) d x \neq 0$, we come to

$$
(x * 1, \phi)=N-\lim _{n \rightarrow \infty}-n \int_{-1}^{1} \tau^{\prime}(y) y \tau(y) d y \int_{-\infty}^{\infty} x \phi(x) d x=0
$$

by noting that

$$
\int_{-1}^{1} \tau^{\prime}(y) y \tau(y) d y=\frac{1}{2} \int_{-1}^{1} \tau^{2}(y) d y
$$

using integration by parts.

Remark 2 In general, the commutative neutrix convolution $x^{r} * x^{s}$ exists (Li et al., in press) for $r, s=0,1,2, \cdots$, and

$$
x^{r} \bar{*} x^{s}=-B(r+1, s+1)\left[x_{+}^{r+s+1}+(-1)^{r+s} x_{-}^{r+s+1}\right]
$$

where $B$ denotes the Beta function.

\section{Fractional Derivative of Distributions}

Let $\mathcal{D}^{\prime}\left(R^{+}\right)$be the subspace of $\mathcal{D}^{\prime}$ with support contained in $R^{+}$. It follows from (Gel'fand \& Shilov, 1964; Li, 2015) that $\Phi_{\lambda}=\frac{x_{+}^{\lambda-1}}{\Gamma(\lambda)} \in \mathcal{D}^{\prime}\left(R^{+}\right)$is an entire function of $\lambda$ on the complex plane, and

$$
\left.\frac{x_{+}^{\lambda-1}}{\Gamma(\lambda)}\right|_{\lambda=-n}=\delta^{(n)}(x) \quad \text { for } \quad n=0,1,2, \cdots
$$

For the functional $\Phi_{\lambda}=\frac{x_{+}^{\lambda-1}}{\Gamma(\lambda)}$, the derivative formula is simpler than that for $x_{+}^{\lambda}$. In fact,

$$
\frac{d}{d x} \Phi_{\lambda}=\frac{d}{d x} \frac{x_{+}^{\lambda-1}}{\Gamma(\lambda)}=\frac{(\lambda-1) x_{+}^{\lambda-2}}{\Gamma(\lambda)}=\frac{x_{+}^{\lambda-2}}{\Gamma(\lambda-1)}=\Phi_{\lambda-1} .
$$

Let $\lambda$ and $\mu$ be arbitrary complex numbers. Then it is easy to show

$$
\Phi_{\lambda} * \Phi_{\mu}=\Phi_{\lambda+\mu}
$$

by equation (4), without any help of analytic continuation mentioned in all current books. 
Let $\lambda$ be an arbitrary complex number and $H$ be a subspace of $\mathcal{D}^{\prime}$ given by

$$
H=\left\{g \in \mathcal{D}^{\prime} \mid g * \Phi_{\lambda} \text { exists }\right\} .
$$

Clearly, $H$ contains $\mathcal{D}^{\prime}\left(R^{+}\right)$as a proper subspace since Definition 1.6 generalizes Definition 1.2.

Let $g(x)$ be the distribution in $H$. We define the primitive of order $\lambda$ of $g$ as the convolution given by Definition 1.6 in the distributional sense:

$$
g_{\lambda}(x)=g(x) * \frac{x_{+}^{\lambda-1}}{\Gamma(\lambda)}=g(x) \approx \Phi_{\lambda} .
$$

Note that the convolution on the right-hand side is well defined since $g$ is in $H$.

Thus equation (6) with various $\lambda$ will not only give the fractional derivatives, but also the fractional integrals of $g(x) \in H$ when $\lambda \notin Z$, and it reduces to integer-order derivatives or integrals when $\lambda \in Z$. We shall define the convolution

$$
g_{-\lambda}=g(x) \bar{*} \Phi_{-\lambda}
$$

as the fractional derivative of the distribution $g(x)$ with order $\lambda$, writing it as

$$
g_{-\lambda}=\frac{d^{\lambda}}{d x^{\lambda}} g
$$

for $\operatorname{Re} \lambda \geq 0$. Similarly, $\frac{d^{\lambda}}{d x^{\lambda}} g$ is interpreted as the fractional integral of order $\lambda$ if $\operatorname{Re} \lambda<0$.

We are now ready to show the following fractional Taylor's expansion in the distributional sense with applications presented in several examples.

Theorem 3.1 Let $\lambda \geq 0$ and $\phi(x) \in C^{\infty}[0, \infty)$ satisfying

$$
\phi(x)=\sum_{k=0}^{\infty} \frac{\phi^{(k)}(0)}{k !} x^{k}
$$

Then the following fractional Taylor's expansion holds

$$
\frac{d^{\lambda}}{d x^{\lambda}} \theta(x) \phi(x)=\sum_{k=0}^{\infty} \frac{\phi^{(k)}(0) x_{+}^{k-\lambda}}{\Gamma(k-\lambda+1)}
$$

where

$$
\theta(x)= \begin{cases}1 & \text { if } x>0 \\ 0 & \text { if } x<0\end{cases}
$$

Proof. Clearly,

$$
\frac{d^{\lambda}}{d x^{\lambda}} \frac{x_{+}^{k}}{\Gamma(k+1)}=\frac{x_{+}^{k-\lambda}}{\Gamma(k-\lambda+1)}
$$

for all $k=0,1,2, \cdots$ and $\lambda \geq 0$ by equation (4).

It follows that

$$
\begin{aligned}
\frac{d^{\lambda}}{d x^{\lambda}} \theta(x) \phi(x) & =\frac{d^{\lambda}}{d x^{\lambda}} \sum_{k=0}^{\infty} \frac{\phi^{(k)}(0)}{k !} x_{+}^{k}=\sum_{k=0}^{\infty} \frac{d^{\lambda}}{d x^{\lambda}} \frac{\phi^{(k)}(0)}{k !} x_{+}^{k} \\
& =\sum_{k=0}^{\infty} \frac{\phi^{(k)}(0) x_{+}^{k-\lambda}}{\Gamma(k-\lambda+1)}
\end{aligned}
$$

which clearly converges in the distributional sense, as $\sum_{k=0}^{\infty} \frac{\phi^{(k)}(0)}{k !} x^{k}$ converges to $\phi(x)$ as well as two Stirling's formulas below

$$
\begin{aligned}
& \Gamma(k-\lambda+1) \sim \sqrt{2 \pi(k-\lambda)}\left(\frac{k-\lambda}{e}\right)^{k-\lambda}, \text { and } \\
& k ! \sim \sqrt{2 \pi k}\left(\frac{k}{e}\right)^{k} .
\end{aligned}
$$


In particular, we get for $\lambda=m \in Z^{+}$

$$
\begin{aligned}
\frac{d^{m}}{d x^{m}} \theta(x) \phi(x) & =\sum_{k=0}^{\infty} \frac{\phi^{(k)}(0) x_{+}^{k-m}}{\Gamma(k-m+1)} \\
& =\phi(0) \frac{x_{+}^{-m}}{\Gamma(-m+1)}+\cdots+\phi^{(m-1)}(0) \frac{x_{+}^{-1}}{\Gamma(0)}+\sum_{k=m}^{\infty} \frac{\phi^{(k)}(0) x_{+}^{k-m}}{\Gamma(k-m+1)} \\
& =\phi(0) \delta^{(m-1)}(x)+\cdots+\phi^{(m-1)}(0) \delta(x)+\theta(x) \phi^{(m)}(x)
\end{aligned}
$$

which can be obtained distributionally. Indeed, we have for $\psi \in \mathcal{D}$

$$
\begin{aligned}
& \left(\frac{d}{d x} \theta(x) \phi(x), \psi(x)\right)=-\left(\theta(x) \phi(x), \psi^{\prime}(x)\right)=-\int_{0}^{\infty} \phi(x) \psi^{\prime}(x) d x \\
& =-\left.\phi(x) \psi(x)\right|_{0} ^{\infty}+\int_{0}^{\infty} \phi^{\prime}(x) \psi(x) d x=\left(\phi(0) \delta(x)+\theta(x) \phi^{\prime}(x), \psi(x)\right),
\end{aligned}
$$

which implies

$$
\frac{d}{d x} \theta(x) \phi(x)=\phi(0) \delta(x)+\theta(x) \phi^{\prime}(x) .
$$

Evidently

$$
\begin{aligned}
\frac{d^{2}}{d x^{2}} \theta(x) \phi(x) & =\frac{d}{d x}\left(\phi(0) \delta(x)+\theta(x) \phi^{\prime}(x)\right) \\
& =\phi(0) \delta^{\prime}(x)+\phi^{\prime}(0) \delta(x)+\theta(x) \phi^{\prime \prime}(x)
\end{aligned}
$$

by recursion and the identity

$$
\phi^{\prime}(x) \delta(x)=\phi^{\prime}(0) \delta(x)
$$

This claims in general

$$
\frac{d^{m}}{d x^{m}} \theta(x) \phi(x)=\phi(0) \delta^{(m-1)}(x)+\cdots+\phi^{(m-1)}(0) \delta(x)+\theta(x) \phi^{(m)}(x) .
$$

This completes the proof of Theorem 3.1.

Example 1 Let $\lambda \geq 0$ and $\theta(x)$ is given in Theorem 3.1. Then

$$
\frac{d^{\lambda}}{d x^{\lambda}} \theta(x)(1+x) e^{x}=\sum_{k=0}^{\infty} \frac{(k+1) x_{+}^{k-\lambda}}{\Gamma(k-\lambda+1)} .
$$

In particular,

$$
\frac{d^{\frac{1}{2}}}{d x^{\frac{1}{2}}} \theta(x)(1+x) e^{x}=\sum_{k=0}^{\infty} \frac{4^{k}(k+1) ! x_{+}^{k-1 / 2}}{(2 k) ! \sqrt{\pi}} .
$$

Proof. Indeed, $\phi(x)=(1+x) e^{x} \in C^{\infty}(R)$ and

$$
\begin{aligned}
(1+x) e^{x} & =\sum_{n=0}^{\infty} \frac{x^{n}}{n !}+\sum_{n=0}^{\infty} \frac{x^{n+1}}{n !} \\
& =1+\sum_{n=1}^{\infty}\left(\frac{1}{n !}+\frac{1}{(n-1) !}\right) x^{n} \\
& =\sum_{n=0}^{\infty} \frac{n+1}{n !} x^{n} .
\end{aligned}
$$

This implies that

$$
\left.\left((1+x) e^{x}\right)^{(n)}\right|_{x=0}=n+1 .
$$

By equation (7), we get equation (8). Using the identity

$$
\Gamma(k+1 / 2)=\frac{(2 k) !}{4^{k}(k) !} \sqrt{\pi},
$$


we show that

$$
\frac{d^{\frac{1}{2}}}{d x^{\frac{1}{2}}} \theta(x)(1+x) e^{x}=\sum_{k=0}^{\infty} \frac{4^{k}(k+1) ! x_{+}^{k-1 / 2}}{(2 k) ! \sqrt{\pi}} .
$$

Example 2 Let $0<\lambda<1$ and $\theta(x)$ is given in Theorem 3.1. Then

$$
\frac{d^{\lambda}}{d x^{\lambda}} \theta(x) \sinh x=\sum_{n=0}^{\infty} \frac{x_{+}^{1+2 n-\lambda}}{\Gamma(2+2 n-\lambda)} .
$$

Proof. Applying

$$
\sinh x=\sum_{n=0}^{\infty} \frac{x^{2 n+1}}{(2 n+1) !}
$$

for all $x \in R$, we derive that

$$
\left.(\sinh x)^{(2 n+1)}\right|_{x=0}=1, \quad \text { and }\left.\quad(\sinh x)^{(2 n)}\right|_{x=0}=0
$$

for $n=0,1,2, \cdots$. By equation (7), we come to

$$
\begin{aligned}
\frac{d^{\lambda}}{d x^{\lambda}} \theta(x) \sinh x & =\sum_{k=0}^{\infty} \frac{\sinh ^{(k)}(0) x_{+}^{k-\lambda}}{\Gamma(k-\lambda+1)} \\
& =\sum_{n=0}^{\infty} \frac{x_{+}^{1-\lambda+2 n}}{\Gamma(2-\lambda+2 n)} .
\end{aligned}
$$

In particular,

$$
\frac{d^{\frac{1}{2}}}{d x^{\frac{1}{2}}} \theta(x) \sinh x=\sum_{n=0}^{\infty} \frac{4^{2 n+1}(2 n+1) ! x_{+}^{1 / 2+2 n}}{(4 n+2) ! \sqrt{\pi}} .
$$

Similarly, we get

$$
\frac{d^{\lambda}}{d x^{\lambda}} \theta(x) \cosh x=\sum_{n=0}^{\infty} \frac{x_{+}^{-\lambda+2 n}}{\Gamma(1-\lambda+2 n)}
$$

for $0<\lambda<1$ by using the formula

$$
\cosh x=\sum_{n=0}^{\infty} \frac{x^{2 n}}{(2 n) !}
$$

for all $x \in R$.

In particular,

$$
\frac{d^{\frac{1}{2}}}{d x^{\frac{1}{2}}} \theta(x) \cosh x=\sum_{n=0}^{\infty} \frac{4^{2 n}(2 n) ! x_{+}^{-1 / 2+2 n}}{(4 n) ! \sqrt{\pi}} .
$$

Example 3 Let $\lambda>0$ and

$$
g(x)= \begin{cases}-x & \text { if } 0<x<1 \text { and } x \text { is irrational } \\ 0 & \text { otherwise }\end{cases}
$$

Then

$$
\frac{d^{\lambda}}{d x^{\lambda}} g(x)=\frac{(x-1)_{+}^{-\lambda}}{\Gamma(1-\lambda)}+\frac{(x-1)_{+}^{1-\lambda}}{\Gamma(2-\lambda)}-\frac{x_{+}^{1-\lambda}}{\Gamma(2-\lambda)} .
$$

Proof. Clearly,

$$
\begin{aligned}
\left(g^{\prime}(x), \phi(x)\right) & =-\left(g(x), \phi^{\prime}(x)\right)=\int_{0}^{1} x \phi^{\prime}(x) d x \\
& =\phi(1)-\int_{0}^{1} \phi(x) d x \\
& =(\delta(x-1), \phi(x))+\int_{-\infty}^{\infty}(\theta(x-1)-\theta(x)) \phi(x) d x
\end{aligned}
$$


This infers that

$$
g^{\prime}(x)=\delta(x-1)+\theta(x-1)-\theta(x) .
$$

Hence,

$$
\frac{d^{m}}{d x^{m}} g(x)=\delta^{(m-1)}(x-1)+\delta^{(m-2)}(x-1)-\delta^{(m-2)}(x)
$$

if $m \geq 2$, and

$$
\begin{aligned}
& \frac{d^{\lambda}}{d x^{\lambda}} g(x)=g(x) * \frac{x_{+}^{-\lambda-1}}{\Gamma(-\lambda)}=g(x) * \frac{d^{m}}{d x^{m}} \frac{x_{+}^{m-\lambda-1}}{\Gamma(m-\lambda)} \\
& =\frac{d^{m}}{d x^{m}} g(x) * \frac{x_{+}^{m-\lambda-1}}{\Gamma(m-\lambda)}
\end{aligned}
$$

where $0<m-\lambda<1$.

If $m=1$, then $0<\lambda<1$ and

$$
\begin{aligned}
\frac{d^{\lambda}}{d x^{\lambda}} g(x) & =(\delta(x-1)+\theta(x-1)-\theta(x)) * \frac{x_{+}^{-\lambda}}{\Gamma(1-\lambda)} \\
& =\frac{(x-1)_{+}^{-\lambda}}{\Gamma(1-\lambda)}+\frac{(x-1)_{+}^{1-\lambda}}{\Gamma(2-\lambda)}-\frac{x_{+}^{1-\lambda}}{\Gamma(2-\lambda)} .
\end{aligned}
$$

If $m \geq 2$, then we also have

$$
\begin{aligned}
\frac{d^{\lambda}}{d x^{\lambda}} g(x) & =\left(\delta^{(m-1)}(x-1)+\delta^{(m-2)}(x-1)-\delta^{(m-2)}(x)\right) * \frac{x_{+}^{m-\lambda-1}}{\Gamma(m-\lambda)} \\
& =\frac{(x-1)_{+}^{-\lambda}}{\Gamma(1-\lambda)}+\frac{(x-1)_{+}^{1-\lambda}}{\Gamma(2-\lambda)}-\frac{x_{+}^{1-\lambda}}{\Gamma(2-\lambda)} .
\end{aligned}
$$

The following theorem can be found in (Li et al., 2017).

Theorem 3.2 Let $g(x)$ be given in $\mathcal{D}^{\prime}\left(R^{+}\right)$and $f(x)$ be unknown in $\mathcal{D}^{\prime}\left(R^{+}\right)$. Then the generalized Abel's integral equation given by

$$
g(x)=\frac{1}{\Gamma(1-\alpha)} \int_{0}^{x} \frac{f(\zeta)}{(x-\zeta)^{\alpha}} d \zeta
$$

has the solution

$$
f(x)=g(x) * \Phi_{\alpha-1}
$$

where $\alpha$ is any real number. In particular, if $-m<\alpha<-m+1$ for $m \in Z^{+}$then

$$
f(x)=g^{(m+1)}(x) * \frac{x_{+}^{\alpha+m-1}}{\Gamma(\alpha+m)} .
$$

As an application of fractional derivative in distribution, we present the following theorem which converts Poisson's integral equation to the generalized Abel's integral equation. Hence we are able to solve it by Theorem 3.2.

Theorem 3.3 Let $\lambda>0$. Then the following Poisson's integral equation

$$
\int_{0}^{\pi / 2} f(r \cos \omega) \sin ^{2 \lambda+1} \omega d \omega=\theta(r) g(r)
$$

has the solution

$$
f(\sqrt{t})=\frac{2 \sqrt{t}}{\Gamma(\lambda+1)} \frac{d^{\lambda}}{d t^{\lambda}} \theta(t) t^{\lambda+1 / 2} g(\sqrt{t})
$$

where $f$ is unknown, and $g$ is given in $\mathcal{D}^{\prime}\left(R^{+}\right)$.

Proof. Performing the substitution $x=r \cos \omega$ we convert equation (9) into

$$
\int_{0}^{r} f(x)\left(1-\frac{x^{2}}{r^{2}}\right)^{\lambda} d x=\theta(r) r g(r)
$$


setting

we come to

$$
y=\frac{1}{r^{2}}
$$

$$
\int_{0}^{1 / \sqrt{y}} f(x)\left(1-y x^{2}\right)^{\lambda} d x=\theta(y) \frac{1}{\sqrt{y}} g\left(\frac{1}{\sqrt{y}}\right),
$$

which can be changed to

$$
\int_{0}^{1 / \sqrt{y}} f(x)\left(\frac{1}{y}-x^{2}\right)^{\lambda} d x=\theta(y) \frac{1}{y^{\lambda+1 / 2}} g\left(\frac{1}{\sqrt{y}}\right) .
$$

Making the substitutions

$$
\tau=x^{2}, \quad t=\frac{1}{y}
$$

we get Abel's integral equation

$$
\int_{0}^{t}(t-\tau)^{\lambda} \frac{f(\sqrt{\tau})}{\sqrt{\tau}} d \tau=2 \theta(t) t^{\lambda+1 / 2} g(\sqrt{t})
$$

which has the solution

$$
f(\sqrt{t})=\frac{2 \sqrt{t}}{\Gamma(\lambda+1)} \frac{d^{\lambda}}{d t^{\lambda}} \theta(t) t^{\lambda+1 / 2} g(\sqrt{t})
$$

by Theorem 3.2. This completes the proof of Theorem 3.3.

Remark 3 We would like to add that all the derivatives in Theorem 3.3 are in the distributional sense and they may not exist classically, as the fractional derivatives of distributions are more general. Here is an example to illustrate this. Assume that $g(\sqrt{t})=t_{+}^{-2.6}$ and $\lambda=1 / 2$. Then $\frac{d^{1 / 2}}{d t^{1 / 2}} \theta(t) t_{+}^{-1.6}$ only exists distributionally, but not classically.

Example 4 The following Poisson's integral equation

$$
\int_{0}^{\pi / 2} f(r \cos \omega) \sin ^{2} \omega d \omega=\theta(r) e^{r^{2}}
$$

has the solution

$$
f(t)=\frac{16}{\pi} \sum_{k=0}^{\infty} \frac{4^{k}(k+1) !(k+1) t_{+}^{2 k+2}}{(2 k+2) !} .
$$

Proof. Indeed, we have $\lambda=1 / 2$ and $g(r)=\theta(r) e^{r^{2}}$. By Theorem 3.3, we come to

$$
f(\sqrt{t})=\frac{2 \sqrt{t}}{\Gamma(1 / 2+1)} \frac{d^{1 / 2}}{d t^{1 / 2}} \theta(t) t e^{t} .
$$

Clearly,

$$
t e^{t}=\sum_{k=0}^{\infty} \frac{k t^{k}}{k !}
$$

which implies that

$$
\left.\left(t e^{t}\right)^{(k)}\right|_{x=0}=k
$$

for $k=0,1,2 . \cdots$. Therefore, we have by equation (7)

$$
\frac{d^{1 / 2}}{d t^{1 / 2}} \theta(t) t e^{t}=\sum_{k=-1}^{\infty} \frac{(k+1) t_{+}^{1-1 / 2+k}}{\Gamma(2-1 / 2+k)}=t_{+}^{-1 / 2} \sum_{k=0}^{\infty} \frac{(k+1) t_{+}^{k+1}}{\Gamma(k+3 / 2)} .
$$

This infers that

$$
f(\sqrt{t})=\frac{4}{\sqrt{\pi}} \sum_{k=0}^{\infty} \frac{4^{k+1}(k+1) !(k+1)}{(2 k+2) ! \sqrt{\pi}} t_{+}^{k+1}=\frac{16}{\pi} \sum_{k=0}^{\infty} \frac{4^{k}(k+1) !(k+1)}{(2 k+2) !} t_{+}^{k+1} .
$$

So that

$$
f(t)=\frac{16}{\pi} \sum_{k=0}^{\infty} \frac{4^{k}(k+1) !(k+1)}{(2 k+2) !} t_{+}^{2 k+2} .
$$


Example 5 Poisson's integral equation

$$
\int_{0}^{\pi / 2} f(r \cos \omega) \sin ^{2 \lambda+1} \omega d \omega=\theta(r) g(r)
$$

has the solution

$$
f(t)=\frac{2 t^{2}}{\Gamma(\lambda+1)} \sum_{k=0}^{\infty} \frac{\Gamma(k / 2+1 / 2+\lambda+1) g^{(k)}(0)}{k ! \Gamma(k / 2+1 / 2+1)} t_{+}^{k} .
$$

where $\lambda>0$ and

$$
g(t)=\sum_{k=0}^{\infty} \frac{g^{(k)}(0)}{k !} t^{k}
$$

Proof. By Theorem 3.3, we come to

$$
\begin{aligned}
f(\sqrt{t}) & =\frac{2 \sqrt{t}}{\Gamma(\lambda+1)} \frac{d^{\lambda}}{d t^{\lambda}} \theta(t) t^{\lambda+1 / 2} g(\sqrt{t}) \\
& =\frac{2 \sqrt{t}}{\Gamma(\lambda+1)} \frac{d^{\lambda}}{d t^{\lambda}} \sum_{k=0}^{\infty} \frac{g^{(k)}(0)}{k !} t_{+}^{k / 2+1 / 2+\lambda} \\
& =\frac{2 \sqrt{t}}{\Gamma(\lambda+1)} \sum_{k=0}^{\infty} \frac{g^{(k)}(0)}{k !} \frac{d^{\lambda}}{d t^{\lambda}} l_{+}^{k / 2+1 / 2+\lambda} .
\end{aligned}
$$

Clearly,

$$
\begin{aligned}
\frac{d^{\lambda}}{d t^{\lambda}} t_{+}^{k / 2+1 / 2+\lambda} & =\frac{d^{\lambda}}{d t^{\lambda}} \Gamma(k / 2+1 / 2+\lambda+1) \frac{t_{+}^{k / 2+1 / 2+\lambda}}{\Gamma(k / 2+1 / 2+\lambda+1)} \\
& =\Gamma(k / 2+1 / 2+\lambda+1) \frac{t_{+}^{k / 2+1 / 2}}{\Gamma(k / 2+1 / 2+1)} .
\end{aligned}
$$

Therefore,

$$
f(\sqrt{t})=\frac{2 t}{\Gamma(\lambda+1)} \sum_{k=0}^{\infty} \frac{\Gamma(k / 2+1 / 2+\lambda+1) g^{(k)}(0)}{k ! \Gamma(k / 2+1 / 2+1)} t_{+}^{k / 2},
$$

which implies that

$$
f(t)=\frac{2 t^{2}}{\Gamma(\lambda+1)} \sum_{k=0}^{\infty} \frac{\Gamma(k / 2+1 / 2+\lambda+1) g^{(k)}(0)}{k ! \Gamma(k / 2+1 / 2+1)} t_{+}^{k} .
$$

Example 6 Let $\lambda>0$ with $\lambda \neq 1,2, \cdots$, and

$$
\phi(x)=\sum_{k=0}^{\infty} \frac{\phi^{(k)}(0)}{k !} x^{k} .
$$

Then the following one-term fractional differential equation

$$
\frac{d^{\lambda}}{d x^{\lambda}} y(x)=\theta(x) \phi(x)
$$

with the initial conditions

$$
y^{(0)}(0)=0, y^{(1)}(0)=0, \cdots, y^{(\lfloor\lambda])}(0)=0
$$

has the solution

$$
y(x)=\sum_{k=0}^{\infty} \frac{\phi^{(k)}(0)}{\Gamma(\lambda+k+1)} x_{+}^{k+\lambda} .
$$

Proof. Assume

$$
y(x)=\sum_{k=0}^{\infty} \frac{y^{(k)}(0)}{k !} x_{+}^{\lambda+k} .
$$


Then using

we convert equation (11) into

$$
\frac{d^{\lambda}}{d x^{\lambda}} \frac{x_{+}^{\lambda+k}}{\Gamma(\lambda+k+1)}=\frac{x_{+}^{k}}{\Gamma(k+1)},
$$

$$
\sum_{k=0}^{\infty} \frac{y^{(k)}(0)}{k !} \frac{\Gamma(\lambda+k+1)}{\Gamma(k+1)} x_{+}^{k}=\sum_{k=0}^{\infty} \frac{\phi^{(k)}(0)}{k !} x_{+}^{k}
$$

and comparison of the coefficients of both series gives

$$
y^{(k)}(0)=\frac{k ! \phi^{(k)}(0)}{\Gamma(\lambda+k+1)} .
$$

Finally, we turn our attention to computing fractional derivative of $x_{-}^{s}$ (note that the support is not in $R^{+}$) for $s=0,1,2, \cdots$, based on the following formulas presented in (Li et al., in press)

$$
\begin{aligned}
& x_{+}^{\lambda} \bar{*} x_{-}^{s}=(-1)^{s+1} B(\lambda+1, s+1) x_{+}^{\lambda+s+1}, \\
& x_{+}^{\lambda} \bar{*} x^{s}=0
\end{aligned}
$$

for $\lambda \neq 0, \pm 1, \pm 2, \cdots$ and $s=0,1,2, \cdots$.

Theorem 3.4 Let $\lambda>0$ and $\lambda \neq 1,2, \cdots$. Then

$$
\frac{d^{\lambda}}{d t^{\lambda}} x_{-}^{s}=(-1)^{s+1} \frac{s !}{\Gamma(-\lambda+s+1)} x_{+}^{-\lambda+s} .
$$

Proof. Clearly, we have by equation (12)

$$
\begin{aligned}
\frac{d^{\lambda}}{d t^{\lambda}} x_{-}^{s} & =x_{-}^{s} \mp \frac{x_{+}^{-\lambda-1}}{\Gamma(-\lambda)}=(-1)^{s+1} \frac{B(-\lambda, s+1)}{\Gamma(-\lambda)} x_{+}^{-\lambda+s} \\
& =(-1)^{s+1} \frac{s !}{\Gamma(-\lambda+s+1)} x_{+}^{-\lambda+s} .
\end{aligned}
$$

This completes the proof of Theorem 3.4.

Remark 4 We would like to mention that there is another approach to proving equation (14) using equation (13). Indeed,

$$
\frac{x_{+}^{-\lambda-1}}{\Gamma(-\lambda)} \bar{*} x^{s}=\left[x_{+}^{s}+(-1)^{s} x_{-}^{s}\right] * \frac{x_{+}^{-\lambda-1}}{\Gamma(-\lambda)}=0
$$

which implies that

$$
\begin{aligned}
x_{-}^{s} * \frac{x_{+}^{-\lambda-1}}{\Gamma(-\lambda)} & =(-1)^{s+1} x_{+}^{s} * \frac{x_{+}^{-\lambda-1}}{\Gamma(-\lambda)}=(-1)^{s+1} s ! \frac{x_{+}^{s}}{\Gamma(s+1)} \approx \frac{x_{+}^{-\lambda-1}}{\Gamma(-\lambda)} \\
& =(-1)^{s+1} \frac{s !}{\Gamma(-\lambda+s+1)} x_{+}^{-\lambda+s}
\end{aligned}
$$

by equation (5).

\section{Conclusion}

In this paper, we mainly provide a counterexample to show that two definitions for defining distributional convolution are not equivalent, and further study fractional calculus of distributions based on our newly defined convolution. We present a fractional Taylor's expansion with several applications to solving Poisson's integral equations and fractional differential equation in the generalized sense, which cannot be achieved classically. A challenge problem is how to solve the generalized Abel's integral equation for all $\alpha \in R$

$$
g(x)=\frac{1}{\Gamma(1-\alpha)} \int_{-\infty}^{x} \frac{f(\zeta)}{(x-\zeta)^{\alpha}} d \zeta
$$

where $g \in \mathcal{D}^{\prime}(R)$ is given and $f$ is unknown. The authors welcome and appreciate any discussion from interested readers. 


\section{Acknowledgements}

This work is partially supported by the Natural Sciences and Engineering Research Council of Canada and Brandon University Research Grant. The authors are grateful to the reviewers and editor for the careful reading of the article along with several productive suggestions, which have improved its quality.

\section{References}

Aguirre, M., \& Li, C. (2007). The distributional products of particular distributions. Appl. Math. Comput. 187, 20-26. https://doi.org/10.1016/j.amc.2006.08.098

Cheng, L., \& Li, C. (1991). A commutative product of distributions on $R^{m}$. Math. Nachr. 151, 345-355. https://doi.org/10.1002/mana.19911510124

Fisher, B. (1975). A result of the convolution of distributions. Proc. Edinburgh Math. Soc. 19, 393-395. https://doi.org/10.1002/mana.19821060123

Fisher, B. (1982). On defining the convolution of distributions. Math. Nachr. 106, 261-268. https://doi.org/10.1002/mana.19821060123

Fisher, B. (1987). Neutices and the convolution of distributions. Univ. u Novom Sadu Zb. Rad. Prirod. - Mat. Fak. Ser. Mat. 17, 119-135.

Fisher, B., \& Al-Sirehy, F. (2015). On the convolution and neutrix convolution of the functions $\sinh ^{-1} x$ and $x^{r}$. Sarajevo Journal of Mathematics, 11, 37-48. https://doi.org/10.5644/SJM.11.1.03

Fisher, B., \& Li, C. (1993). A commutative neutrix convolution product of distributions. Univ. u Novom Sadu Zb. Rad. Prirod. - Mat. Fak. Ser. Mat. 23, 13-27.

Fisher, B., \& Kuribayashi, Y. (1987). Neutrices and the Beta function. Rostock. Math. Kolloq. 32, 56-66.

Fisher, B., \& Ozcag, E. (2012). Some results on the neutrix composition of the delta function. Filomat 26, 1247-1256. https://doi.org/10.2298/FIL1206247F

Fisher, B., Ozcag, E., \& Al-Sirehy, F. (2017). On the Composition and Neutrix Composition of the Delta Function and the Function $\cosh ^{-1}\left(|x|^{1 / r}+1\right)$. Int. J. Anal. Appl. 13, 161-169.

Fisher, B., \& Taş. K. (2005). The convolution of functions and distributions. J. Math. Anal. Appl. 306, 364-374. https://doi.org/10.1016/j.jmaa.2005.01.004

Gel'fand, I. M., \& Shilov, G. E. (1964). Generalized functions. Vol I, Academic Press, New York.

Jones, D. S. (1973). The convolution of generalized functions. Quart. J. Math. Oxford Ser. 24, 145-163. https://doi.org/10.1093/qmath/24.1.145

Kilbas, A. A., Srivastava, H. M., \& Trujillo, J. J. (2016) Theory and Applications of Fractional Differential Equations. Elsevier, New York.

Lazarova, L., Jolevska-Tuneska, B., Akturk, I, \& Ozcag, E. (2016) Note on the Distribution Composition $\left(x_{+}^{\mu}\right)^{\lambda}$. Bull. Malaysian Math. Soc. https://doi.org/10.1007/s40840-016-0342-2

Li, C. (2005). The products on the unit sphere and even-dimension spaces. J. Math. Anal. Appl. 305, 97-106. https://doi.org/10.1016/j.jmaa.2004.10.031

$\mathrm{Li}, \mathrm{C}$. (2007a). A review on the products of distributions. Chapter in Mathematical Methods in Engineering, Springer, 71-96. https://doi.org/10.1007/978-1-4020-5678-9

$\mathrm{Li}, \mathrm{C}$. (2007b). Several results on the commutative neutrix product of distributions. Integral transforms Spec. Funct. 18, 559-568. https://doi.org/10.1080/10652460701366169

Li, C. (2015). Several results of fractional derivatives in $\mathcal{D}^{\prime}\left(R^{+}\right)$. Fractional Calculus and Applied Analysis, 18, 192-207. https://doi.org/10.1515/fca-2015-0013

Li, C., Clarkson K., \& Patel, V. (in press). The Convolution and Fractional Derivative of Distributions. Advances in Analysis.

Li, C., \& Li, C. P. (2014). On defining the distributions $(\delta)^{k}$ and $\left(\delta^{\prime}\right)^{k}$ by fractional derivatives. Appl. Math. Comput. 246, 502-513. https://doi.org/10.1016/j.amc.2014.08.068

Li, C., \& Li, C. P. (2017). Remarks on fractional derivatives of distributions. Tbilisi Mathematical Journal, 10, 1-18. https://doi.org/10.1515/tmj-2017-0001 
Li, C., Li, C. P., Kacmar, B., Lacroix, R., \& Tilbury, K. (2017). The Abel Integral Equations in Distribution. Advances in Analysis, 2, 88-104. https://doi.org/10.22606/aan.2017.22003

Ozcag, E., Lazarova, L., \& Jolevska-Tuneska, B. (2016). Defining Compositions of $x_{+}^{\mu},|x|^{\mu}, x^{-s}$ and $x^{-s} \ln |x|$ as Neutrix Limit of Regular Sequences. Commun. Math. Stat. 4, 63-80.

van der Corput, J. G. (1959-60). Introdution to the neutrix calculus. J. Analyse Math. 7, 291-398. https://doi.org/10.1007/BF02787689

\section{Copyrights}

Copyright for this article is retained by the author(s), with first publication rights granted to the journal.

This is an open-access article distributed under the terms and conditions of the Creative Commons Attribution license (http://creativecommons.org/licenses/by/4.0/). 